\title{
Evaluation of edge cracks in cross-ply laminates using image correlation and thermoelastic stress analysis
}

\author{
G.P. Battams ${ }^{a}$, J.M. Dulieu-Barton ${ }^{b}$ and S.W. Boyd ${ }^{c}$
}

School of Engineering Sciences, University of Southampton, Southampton, SO17 1BJ, UK

âG.Battams@soton.ac.uk, bjanice@soton.ac.uk, 'S.W.Boyd@soton.ac.uk

Keywords: Edge cracks, cross ply polymer composite laminate, notched, GFRP, TSA, DIC, thermoelastic stress analysis (TSA), digital image correlation (DIC).

\begin{abstract}
The paper describes initial work on using 2D digital image correlation (DIC) and thermoelastic stress analysis (TSA) to obtain data from edge cracks in cross-ply laminates. It is demonstrated that detailed data related to the crack tip stresses can be obtained using TSA. The work reveals some of the limitations experienced when using DIC in applications where high spatial resolution is required. A detailed discussion is provided along with an outline for future work.
\end{abstract}

\section{Introduction}

The increasing use of composites in many crucial engineering applications has placed greater demand on techniques to assess stress concentrations. Damage accumulation in metals is often localised to cracks and can be predicted using various fracture mechanics approaches [1]. In comparison, laminated polymer composite materials display many forms of damage which interact with each another during initiation and propagation. The mechanisms are also dependent on the constituent material and laminate stacking sequence. Matrix cracking is prominent in $90^{\circ}$ layers when loaded in tension and occurs well below the failure load of the laminate [2]. Such cracking has a relatively small affect on the stiffness of a structure but does initiate other types of damage such as fibre breakage and delamination. Therefore it is necessary to apply experimental techniques to provide enhanced understanding of the failure mechanisms

Strain measurements using strain gauges or extensometers are useful for obtaining point readings but only provide information at discrete points. Therefore, for complex components full field techniques are more applicable, especially for identification of damage initiation. Two full field techniques that are commonly used are Digital Image Correlation (DIC) and Thermoelastic Stress Analysis (TSA). TSA has been used in relatively few studies on notched or cracked polymer composite specimens [3,4]. Optical techniques have been used extensively in many materials including metals, ceramics and composites [5]. However, TSA and DIC have not been used in conjunction to obtain data relating to the stress and strain in the component. In the present paper the two techniques will be used to examine the stress/strain distribution produced around an edge notch in a composite specimen. The edge notch provides challenges for both techniques in terms of spatial and temporal resolution. The purpose of the paper is to apply the TSA and DIC techniques to an edge notch in a polymer composite component and to identify the advantages and limitations of using each technique in this application. The motivation is to establish a means of assessing the failure mechanisms at the notch. The failure occurs rapidly and therefore a high speed camera is used with the DIC. It is shown that the TSA provides an excellent validation tool for the DIC. 


\section{Experimental Techniques}

TSA is a well established technique for providing full-field stress data for orthotropic composite materials [3, 4]. The underlying theory is presented in the review [3]. For an orthotropic material such as a laminated polymer composite the temperature change, $\Delta T$, is related to the change in stress as follows [4]:

$$
\Delta T=-\frac{T}{\rho C_{P}}\left(\alpha_{1} \Delta \sigma_{1}+\alpha_{2} \Delta \sigma_{2}\right)
$$

where $C_{p}$ is the specific heat at constant pressure, $T$ is the absolute temperature on the surface, $\alpha$ denotes the coefficient of thermal expansion and $\sigma$ is the direct stress. The subscripts 1 and 2 denote the principal material directions.

The infra-red system used in this work is the Silver $480 \mathrm{M}$ manufactured by Cedip and has a maximum frame rate of $383 \mathrm{~Hz}$ at the full resolution of $320 \times 256$ pixels. The system is radiometrically calibrated and can deliver values of $\Delta T$ directly. In the present work the data will be presented in the form of $\Delta T / T$ to enable data to be compared to other data taken at different room temperatures and also to eliminate the effect of any localised heating close to the damage site. The measured data is $T$, with $\Delta T$ values obtained through performing TSA. This uses the lock-in function, requiring a load reference signal from the test machine used to load the specimens.

Digital Image correlation (DIC) is a technique that utilises standard white light cameras and the contrast of the specimen surface. The correlation was conducted using proprietary software: DaVis by LA Vision. Their correlation algorithm divides the imaged area into a grid of interrogation cells and tracks the movement of each cell in the images collected as a structural test progresses. To enable correlation between the cells, a random surface pattern must be used that has well contrasting pixel grayscale levels; this is achieved using spray paint. The images from the deformed and reference states are correlated, and the displacements obtained. Subsequently the strains are obtained by taking the displacement at the centre of the cell and differentiating over a gauge length defined by the distance between the centres of two adjacent cells. In this paper a single high speed Redlake Motionpro X-3 plus digital camera is used to obtain in-plane 2D deformations and strains. The camera has a maximum frame rate of $2000 \mathrm{~Hz}$ at a resolution of $1280 \times 1024$; higher rates can be achieved if the number of vertical pixels is reduced. The camera stores data internally and has a maximum storage size of $8 \mathrm{~GB}$. The data collection is therefore a compromise between frame-rate, spatial resolution and available recording time.

\section{Specimen manufacture and initial tests}

A $[90,0,90,0]_{S}$ sheet of material was produced using Primco UD001/00 unidirectional glass-epoxy pre-impregnated material. The laminate was cured using an autoclave with an applied pressure of 4 bar at $125^{\circ} \mathrm{C}$ for one hour. The cured laminate had an approximate thickness of $2 \mathrm{~mm}$. Specimens were cut parallel and perpendicular to the $0^{\circ}$ ply to give specimens with $90^{\circ}$ and $0^{\circ}$ surface layers respectively to specimens of $300 \mathrm{~mm}$ long by $25 \mathrm{~mm}$ wide. Edge notches were created with a fine piercing saw blade at the centre of the gauge length. The notches have an approximate width of 0.25 $\mathrm{mm}$.

Initial tests were carried out to determine the approximate failure load and extension to failure of the specimens. Unnotched specimens were found to fail at $18.5 \mathrm{kN}$ with $5.53 \mathrm{~mm}$ extension, giving a Ultimate Tensile Strength (UTS) of $388 \mathrm{MPa}$. When a notch of $2 \mathrm{~mm}$ was introduced into one side 
of the specimen, the approximate failure load was found to be $13.0 \mathrm{kN}$ at $3.35 \mathrm{~mm}$ extension, giving a critical stress intensity factor of around $34 \mathrm{MPa} / \mathrm{mm}^{1 / 2}[1]$.

Tests were conducted at a ramp rate of $3.33 \times 10^{-3} \mathrm{~m} / \mathrm{s}$ on an Instron 5500 servo-mechanical test machine. During the ramp, white light digital images were collected using the Redlake Motionpro X-3 plus high speed digital camera at a frame rate of $7000 / \mathrm{sec}$. The crack propagated across the width of the specimen in less than a second. Therefore a slower crack growth rate is required if the failure were to be imaged successfully. Decreasing the ramp rate to $0.1 \mathrm{~mm} / \mathrm{min}$ slowed the crack propagation, but not enough for a fully controlled crack growth. The crack did not grow until the critical stress was achieved and then the crack grew rapidly, increasing in length by $2.5 \mathrm{~mm}$ in 0.25 s. Ideally, in the 2D DIC conducted here, the camera would image the deformations at a slower frequency during the initial part of the test to conserve memory and then operate at a higher frequency when the actual failure occurs. However, this camera cannot change frame rate mid test. Furthermore, calculations showed that a wider specimen would be necessary to achieve stable crack growth. It was considered that it is the strain field that occurs prior to crack initiation that is important. Therefore it was decided to concentrate on the imaging of the stress and strain fields of the notched specimens prior to failure rather than during crack growth in the experiments described in this paper.

\section{Thermoelastic stress analysis}

A cyclic load of $2 \mathrm{kN}$ with a frequency $10 \mathrm{~Hz}$ was applied to both specimen types, i.e. one with a $90^{\circ}$ surface ply and the other with a $0^{\circ}$ surface, using an Instron servo-hydraulic test machine. During the tests, mean loads of 2, 3, 4, 6 and $10 \mathrm{kN}$ were used to increase the load level in the specimen to initiate failure whilst keeping the background thermoelastic response at the same level. 1080 consecutive infra-red images were taken using a frame rate of $101 \mathrm{~Hz}$ with an integration time of $1300 \mu \mathrm{s}$. The data were processed so that $\Delta T / T$ was obtained. These are shown in Figure 1 for the $0^{\circ}$ surface ply in and Figure 2 for the $90^{\circ}$ surface ply.

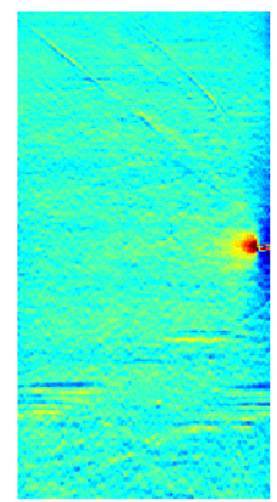

(a)

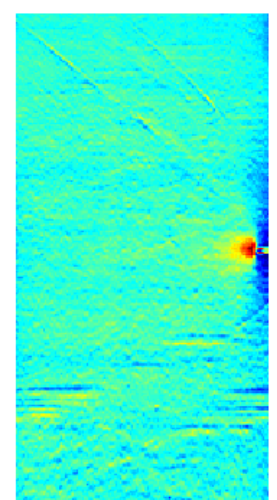

(b)

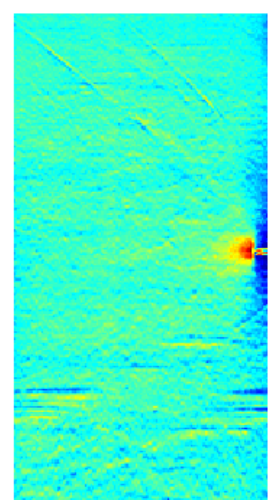

(c)

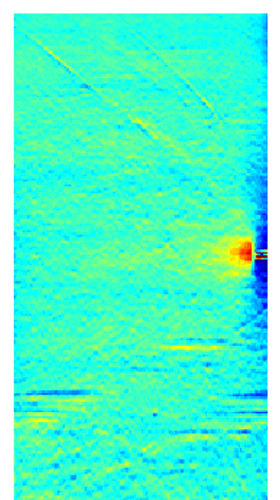

(d)

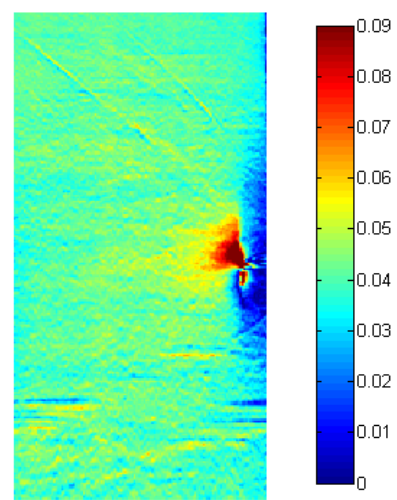

(e)

Figure 1: $\Delta T / T$ for increasing mean load for a $0^{\circ}$ surface ply specimen (a) $2 \mathrm{kN}$ mean load (b) $3 \mathrm{kN} \operatorname{mean} l o a d(c)$ $4 \mathrm{kN}$ mean load (d) $6 \mathrm{kN}$ mean load (e) $10 \mathrm{kN}$ mean load (Image area $=1184 \mathrm{~mm}^{2}$ )

The average $\Delta T / T$ values away from the notch are $0.0375 \pm 0.0035 \mathrm{~K}$ in Figure 1a and 0.0311 $\pm 0.0035 \mathrm{~K}$ in Figure 2a. This shows that the response of the surface ply for the same given applied stress is different corresponding to the findings of [4]. As the mean load increases there is little difference in the average $\Delta T / T$ readings for the $0^{\circ}$ surface ply i.e. $0.0377 \pm 0.0034 \mathrm{~K}$ for the $3 \mathrm{kN}$ load, $0.0382 \pm 0.0034 \mathrm{~K}$ for $4 \mathrm{kN}, 0.0386 \pm 0.0035 \mathrm{~K}$ for $6 \mathrm{kN}$ and $0.0420 \pm 0.0036 \mathrm{~K}$ at the $10 \mathrm{kN}$ load. There is a steady increase in the average value indicating that there is an influence from the damage occurring in the sub surface $90^{\circ}$ ply. This is supported by the readings from the $90^{\circ}$ surface ply: $0.0314 \pm 0.0035 \mathrm{~K}$ for $3 \mathrm{kN}, 0.0339 \pm 0.0036 \mathrm{~K}$ for $4 \mathrm{kN}, 0.0322 \pm 0.0035$ for $6 \mathrm{kN}$ and $0.0271 \pm$ $0.0037 \mathrm{~K}$ for $10 \mathrm{kN}$. As damage evolves with increasing mean load, the $0^{\circ}$ specimen experiences an 
increase in thermoelastic response whereas for the $90^{\circ}$ specimen the response reduces slightly and when gross damage occurs, reduces significantly. The explanation for this is simple; as the $90^{\circ}$ plies fail at lower loads through the creation of transverse cracks, the load carrying capacity is transferred to the $0^{\circ}$ plies and hence the response from one decreases and the other increases. The most interesting and revealing difference in the data is the indication of the presence of the notch. For the specimen with the $0^{\circ}$ surface ply there is a clear stress concentration at the notch identified by an increasing $\Delta T / T$ value with the increasing mean load. This is very similar to what can be seen in an isotropic material with a crack e.g. [4]. In Figure 1e failure has started and there is a clear indication of longitudinal splitting emanating from the notch tip. For the $90^{\circ}$ surface ply, the presence of the notch is indicated only by the reduction in the response above and below the notch where no load is carried. There is a slight increase in response but not at the notch tip. However there are clear indications of transverse cracking between the fibres particularly at the higher mean loads; Figure 2e shows that the transverse cracks have coalesced and the load carrying capability of the ply has diminished significantly. Figures 3 and 4 show plots along a horizontal line through the crack for both specimen types respectively. The peak in response is clearly shown in Figure 3 and is not apparent in Figure 4. There is a difficulty in that the strain in both these specimens is identical and yet the concentration at the crack-tip is not showing in one data set as the response is a result of the surface ply alone.

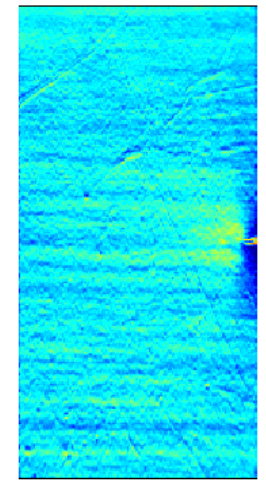

(a)

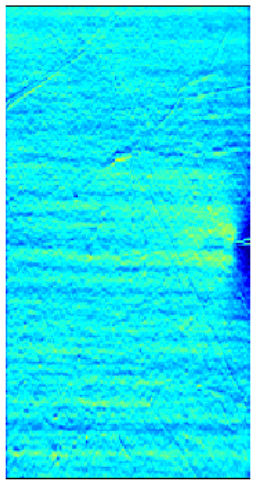

(b)

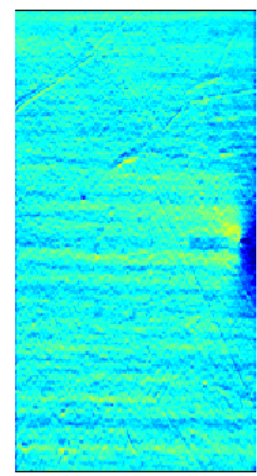

(c)

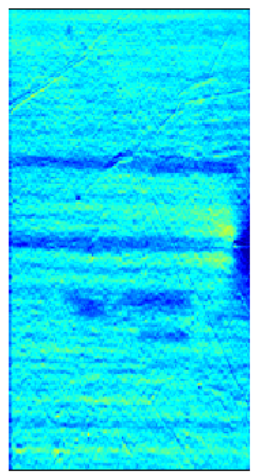

(d)

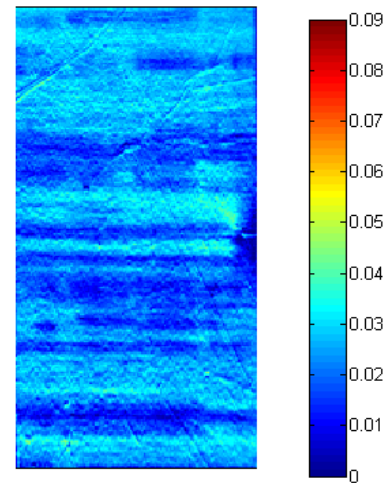

(e)

Figure 2: $\Delta T / T$ for increasing mean load for a $90^{\circ}$ surface ply specimen (a) $2 \mathrm{kN}$ mean load (b) $3 \mathrm{kN}$ mean load (c) $4 \mathrm{kN}$ mean load (d) $6 \mathrm{kN}$ mean load (e) $10 \mathrm{kN}$ mean load (Image area $\left.=1115 \mathrm{~mm}^{2}\right)$

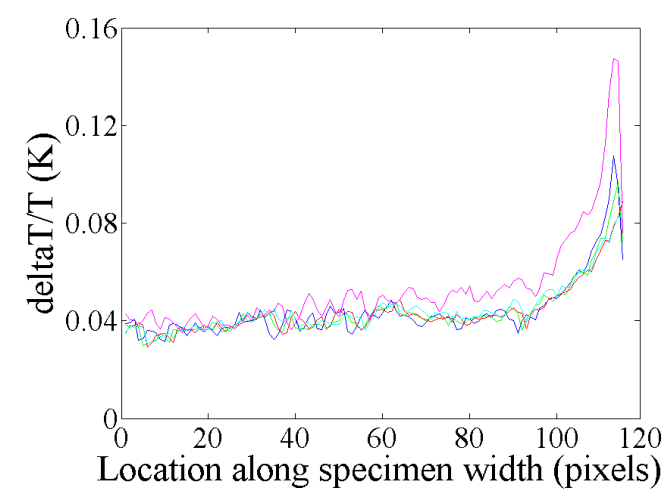

Figure 3: $\Delta T / T$ plot across specimen at notch with increasing mean load for a $0^{\circ}$ surface ply specimen

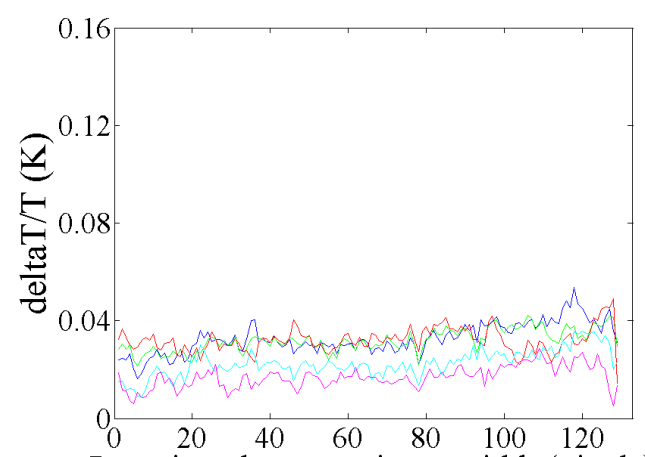

Location along specimen width (pixels)

Figure 4: $\Delta T / T$ plot across specimen at notch with increasing mean load for a $90^{\circ}$ surface ply specimen

\section{Digital image correlation}

$0^{\circ}$ and $90^{\circ}$ surface ply specimens were tested quasi-statically to failure in an Instron 8802 servohydraulic test machine. Two ramp rates were used for the test to minimise overall test time and therefore maximise the image frame rate for the given camera storage capacity. Images were 
captured during the initial ramp of $3.33 \times 10^{-5} \mathrm{~m} / \mathrm{s}$ up to a $3 \mathrm{~mm}$ deflection and the final ramp of $3.33 \times 10^{-6} \mathrm{~m} / \mathrm{s}$ to final failure. The camera was set to a frame rate of $30 \mathrm{~Hz}$ at a resolution of $1080 \mathrm{x}$ 768 pixels giving a raw image spatial resolution of $0.024 \mathrm{~mm}$. Two $1250 \mathrm{~W}$ tungsten lamps were used to illuminate the specimen; load data was simultaneously collected with each image. The speckle pattern was produced by spray paint and the quality of the image was found to be satisfactory based on a histogram of grayscale levels, with a contrast RMS of 176 and an average value of 387 counts. (The manufacturer recommends a minimum of 25 and 80 for RMS and intensity counts respectively.) The images were correlated using the LaVision DaVis DIC software. Initially cell sizes of $64 \times 64$ pixels were used, as was identified in [7] as providing sufficient strain accuracy with the LaVision software. It should be noted that the resolution of the Motion pro camera is significantly less than those used in [7] for image correlation with low speed data capture. The use of a different camera system prompted a further investigation into the effect of cell size. The LaVision correlation algorithm only allows data to be processed using cell sizes of powers of two; all cell sizes from $4 \times 4$ to $64 \times 64$ were examined. All but the $32 \times 32$ and $64 \times 64$ contained large amounts of noise. The results for $32 \times 32$ and $64 \times 64$ for a $0^{\circ}$ and $90^{\circ}$ surface ply are shown in Figures 5 and 6 respectively. The data represent the moment before failure as the image before complete specimen failure is used for the correlation. The $90^{\circ}$ surface ply specimen failed at a load of $11.3 \mathrm{kN}$ and the $0^{\circ}$ surface ply specimen failed at a load of $10.1 \mathrm{kN}$. It would be extremely difficult to capture this data without a high speed camera. For the 64 x 64 cell size which represented $1.52 \mathrm{~mm}^{2}$ on the specimen, the strain is uniform but there is no definition around the notch. The $32 \times 32$ represent a smaller area of $0.76 \mathrm{~mm}^{2}$. The data has better spatial resolution but more noise. A compromise was found by using 64 x 64 cell size with $75 \%$ overlap in the cells; this smoothed the data but enabled much more definition at the notch. The results are shown in Figure 7 for $0^{\circ}$ and $90^{\circ}$ surface plies respectively.

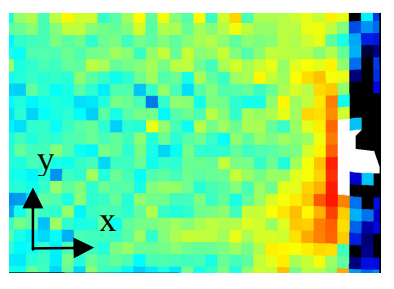

(a)

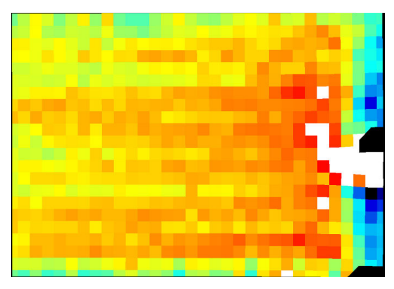

(b)

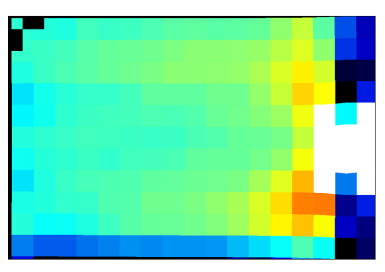

(a)

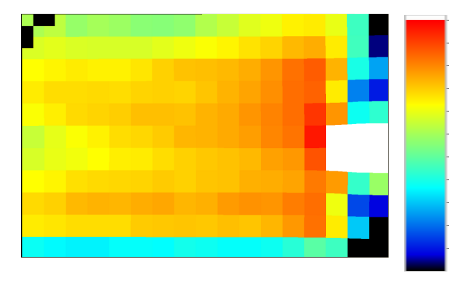

(b)

Figure 5: Strain map $\left(\varepsilon_{y y}\right)$ using a $32 \times 32$ cell size for a $0^{\circ}$ surface ply specimen (a) (Image area $=457$ $\mathrm{mm}^{2}$ ), and a $90^{\circ}$ surface ply specimen (b) (Image area $=462 \mathrm{~mm}^{2}$ )

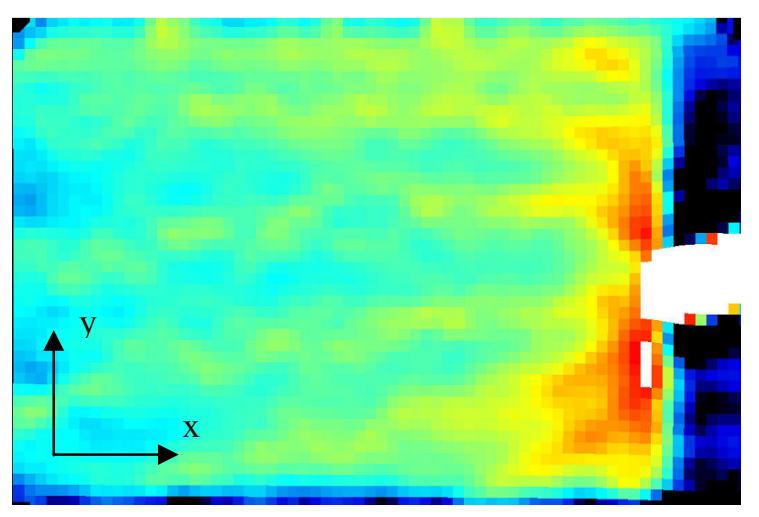

(a)
Figure 6: Strain map $\left(\varepsilon_{y y}\right)$ using a $64 \times 64$ cell size for a $0^{\circ}$ surface ply specimen (a) (Image area $=457$ $\mathrm{mm}^{2}$ ), and a $90^{\circ}$ surface ply specimen (b) (Image area $=462 \mathrm{~mm}^{2}$ )

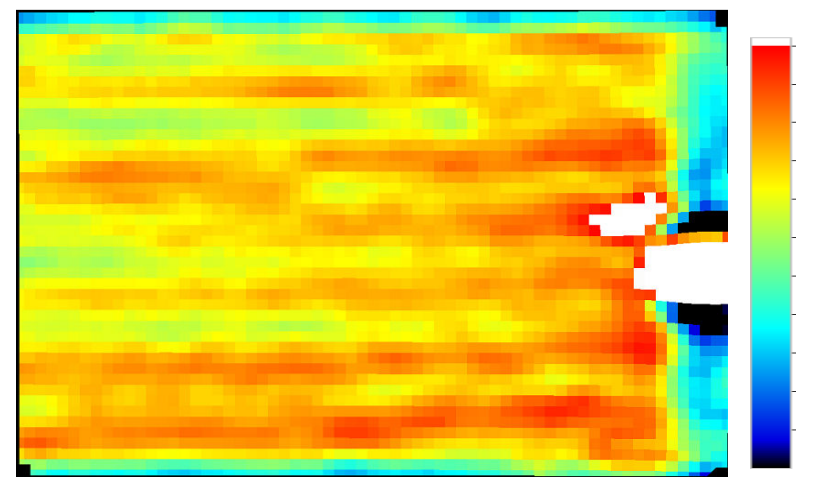

(b)

Figure 7: Strain map $\left(\varepsilon_{y} y\right)$ using a $64 \times 64$ cell size with $75 \%$ overlap for a $0^{\circ}$ surface ply specimen (a) $\left(\right.$ Image area $\left.=457 \mathrm{~mm}^{2}\right)$ and a $90^{\circ}$ surface ply specimen $(b)\left(\right.$ Image area $\left.=462 \mathrm{~mm}^{2}\right)$ 
Figure 7 shows data related to the longitudinal strain. The TSA data is related to a combination of the principal stresses and coefficients of thermal expansion (see Equation (1)). Therefore a direct comparison of the two is not possible. However it is clear that in Figure $7 \mathrm{a}$ there are strain concentrations occurring at the crack and in Figure $7 \mathrm{~b}$ the data is showing overall ply failure. The resolution of the TSA data is far superior but it does not deliver component direct strains. However, a combination of the two techniques could be used to evaluate the material condition and provide better insight into the material failure.

\section{Conclusions and future work}

Some of the limitations and advantages of DIC and TSA have been demonstrated in this paper with several useful properties being confirmed for cross-ply laminates. The decreasing load carrying capacity of a $90^{\circ}$ surface layer has been shown through the formation of transverse cracks and inversely the increased stress and consequently a more dramatic stress concentration effect at the notch with a $0^{\circ}$ surface layer. Spatial resolution and internal camera storage capacity have been found to be a key limitation for the high speed camera system used. However, it has been clearly demonstrated that the high speed DIC can capture the strain state just prior to material failure. A direct comparison of strain derived from the DIC using high resolution cameras at each load step used in the TSA is the next step in the process. By following the same load steps as in the TSA experiments and calibrating the thermoelastic response with this material, the stress patterns could be compared directly.

\section{Acknowledgements}

The work described in this paper is supported by the UK Engineering and Physical Sciences Research Council (Grant number EP/G042403/1) and DSTL under a joint grant managed programme on damage tolerance.

\section{References}

[1] T. L. Anderson: Fracture Mechanics Fundamentals and Applications, (Taylor and Francis Group Publishing, 2005).

[2] J. F. Caron and A. Ehrlacher, Composites Science and Technology Vol. 57 (1997), p. 12611270.

[3] J. Dulieu-Barton and P. Stanley, Journal of Strain Analysis for Engineering Design Vol. 33 (1998), p. 93-104.

[4] R. K. Fruehmann, J. M. Dulieu-Barton and S. Quinn, Journal of Strain Analysis for Engineering Design Vol. 43 (2008), p. 435-450.

[5] M. Grédiac, Composites Part A: Applied Science and Manufacturing Vol. 35 (2004), p. 751761.

[6] M. Zanganeh, R. A. Tomlinson and J. R. Yates, Journal of Strain Analysis for Engineering Design Vol. 43 (2008), p. 529-537.

[7] D. Khennouf, J. Dulieu-Barton, A. R. Chambers, F. J. Lennard and D. D. Eastop, Strain Vol. 46 (2010), p. 19-32. 